\title{
Software de Educação Alimentar e Nutricional para Idosos: Revisão Integrativa da Literatura
}

\author{
Bárbara De Carli Silveira, ${ }^{1}$ Cariza Teixeira Bohrer, ${ }^{2}$ \\ Mariane Rosa, ${ }^{2}$ Loiva Beatriz Dallepiane ${ }^{3}$
}

RESUMO

O objetivo deste trabalho foi identificar os estudos existentes sobre softwares de educação alimentar e nutricional voltados à população idosa por meio de uma revisão integrativa da literatura. Foram incluídos os artigos originais que respondessem à questão norteadora dessa revisão, disponíveis na íntegra, publicados em português, inglês ou espanhol, nos anos de 2009 a 2018 . A busca dos artigos foi realizada nas bases de dados: Medical Literature Analysis and Retrieval System Online (Medline), Literatura Latino-Americana e do Caribe em Ciências da Saúde (Lilacs) e Scopus. A amostra final foi composta por nove artigos, posto que cinco deles tinham como foco a abordagem de Educação Alimentar e Nutricional na promoção da saúde, enquanto quatro estudos focavam no monitoramento de doenças. Com esta revisão observou-se que a educação em saúde, quando associada às tecnologias para a população idosa, em cinco dos artigos selecionados, parece ser uma ferramenta auxiliar para o autocuidado e corresponsabilização do cuidado no que se refere à abordagem de promoção à saúde de educação alimentar e nutricional para a população em estudo.

Palavras-chave: software; educação alimentar e nutricional; idoso.

\section{FOOD AND NUTRITION EDUCATION SOFTWARE FOR THE ELDERLY: INTEGRATIVE LITERATURE REVIEW}

\section{ABSTRACT}

The aim of this work was to identify existing studies on food and nutrition education software aimed at the elderly population through an integrative literature review, which was performed in five steps. Original research that answered the guiding question of this review, available in full, published in Portuguese, English or Spanish, from 2009 to 2018 were included. The search for the articles was performed on the databases: Medical Literature Analysis and Retrieval System Online (Medline), Latin American and Caribbean Health Sciences Literature (Lilacs) and Scopus. The final sample consisted of nine articles, five of which focused on the approach to Food and Nutrition Education in health promotion, whereas four studies focused on disease monitoring. With this review, it was observed that health education, when associated with technologies for the elderly population, five of the selected articles seem to be an auxiliary tool for self-care and co-responsibility of care regarding the approach to health promotion of food education and nutritional for the study population.

Keywords: software; food and nutrition education; elderly.

RECEBIDO EM: 2/10/2019

MODIFICAÇÕES SOLICITADAS EM: 18/2/2021

ACEITO EM: 12/3/2021

\footnotetext{
1 Prefeitura de Novo Tiradentes.

2 Universidade Federal de Santa Maria - Campus Palmeira das Missões. Palmeira das Missões/RS, Brasil.

${ }^{3}$ Autora correspondente. Universidade Federal de Santa Maria (UFSM) - Campus Palmeira das Missões. Av. Independência, 3751 - Vista Alegre, 98300-000. Palmeira das Missões/RS, Brasil. http://lattes.cnpq.br/5570695730349223. https://orcid. org/0000-0003-1331-2882. loiva.dallepiane@hotmail.com
} 


\section{INTRODUÇÃO}

Nos dias atuais há um expressivo aumento no número de idosos, os quais se mostram cada vez mais participativos nas atividades de vida diárias e de socialização. Com isso, esse grupo etário tem sido incentivado a desempenhar novas funções a fim de obter maior satisfação pessoal, o que contribui para sua qualidade de vida, seja para o trabalho ou lazer. Os idosos buscam informar-se sobre as tecnologias atuais para se sentirem inseridos na sociedade (GUIMARÃES, 2017).

No Brasil, entre 2005 e 2015, a proporção de pessoas com mais de 60 anos de idade aumentou acima da média mundial, passando de 9,8\% para 14,3\%. Entre as Unidades da Federação brasileira, em 2015 os valores extremos de idosos na população foram de 8,0\% nos Estados do Amapá e de Roraima, e 17,8\% no Rio Grande do Sul. A estimativa para 2070 é que a população idosa brasileira ultrapasse os $35,0 \%$, sendo, então, superior ao indicado para o conjunto dos países desenvolvidos (IBGE, 2016).

Em virtude do aumento da população idosa, o propósito de envelhecer com maior qualidade de vida torna-se um objetivo a ser alcançado (GUIMARÃES, 2017). Neste contexto, a Educação Alimentar e Nutricional (EAN) constitui-se em um instrumento importante, posto que a EAN se amplia e se consolida como uma ferramenta para orientar escolhas alimentares da população (PADRÃO; AGUIAR; BARÃO, 2017), o que gera informação e conhecimento sobre a alimentação para os diferentes públicos da população, incluindo os idosos.

$\mathrm{Na}$ área da saúde, os aplicativos móveis e a comunicação e a informação via Web, constituem-se em facilitadores da dinâmica do funcionamento dos serviços de saúde, considerando que reduzem ou evitam deslocamentos, diminuem as demandas da rede de saúde, atenuando agravamentos de tratamentos terapêuticos, e ainda podem auxiliar a referência e contrarreferência, aperfeiçoando o vínculo com o sistema de saúde e com um planejamento assistencial de acordo com as demandas do paciente (MENDEZ et al., 2019).

As principais implicações positivas das tecnologias na vida de idoso referem-se à: capacidade de ativação da memória; ampliação do conhecimento; possibilidade de entretenimento e lazer; facilidade na comunicação com amigos e parentes distantes; e melhoria no acesso às informações. Já as principais dificuldades estão relacionadas com o declínio das habilidades físicas e cognitivas decorrentes do envelhecimento, como memória para fixar os métodos de uso das tecnologias; dificuldade de manusear todas essas ferramentas por não serem muito intuitivas e de fácil acesso; e baixa visão, interferindo nas atividades diárias, leitura e sensibilidade à luz (SANTOS; ALMÊDA, 2017).

Para a população idosa, a qual se mostra disponível a buscar novos aprendizados, as Tecnologias de Informação e Comunicação (TICS) podem atuar como um instrumento de autonomia e de estreitamento entre as gerações, de modo a possibilitar a busca e a troca de informações, além de formação ou concretização de uma rede de relacionamentos afetivos, familiares e sociais (GUIMARÃES, 2017). A relação exitosa entre envelhecimento e tecnologia proporciona garantia de acesso, de inclusão social e de ampliação da autonomia dos idosos, posto que

Editora Unijuí - Revista Contexto \& Saúde - ISSN 2176-7114 - v. 21, n. 43, jul./set. 2021 
as mudanças sociais e tecnológicas interferem em diversos aspectos da vida dessa parcela da população (COSTA; BIFANO, 2017).

No estudo de Amorim et al. (2018) foram encontrados 25 aplicativos abrangendo os temas de saúde dos idosos, cuidado de idosos e informações sobre a saúde e o cuidado de idosos. Estes aplicativos são recursos potenciais para o monitoramento, informação, promoção de hábitos saudáveis e prevenção de doenças e agravos em idosos, podendo ser utilizado pelos familiares, cuidadores e profissionais de saúde, impactando na melhoria da qualidade de saúde do idoso.

É neste cenário virtual que a educação alimentar e nutricional se insere na promoção de uma alimentação adequada e saudável. Segundo estudo de Braz e Lopes (2018), os aplicativos móveis de nutrição no Brasil visam, principalmente, ao monitoramento da dieta e da prática de exercícios físicos realizados pelos usuários, seguimento de refeições diárias e controle de ingestão de energia, promovendo hábitos saudáveis.

É apontado que a EAN é uma ferramenta relevante, e que os aplicativos se mostram úteis na relação do idoso, porém pouco se sabe sobre aplicativos de EAN voltados para o idoso, sendo este, portanto, o objetivo do trabalho.

\section{METODOLOGIA}

Trata-se de uma revisão integrativa da literatura; uma metodologia que possibilita ao pesquisador sumarizar o que se sabe sobre determinado assunto e reconhecer lacunas no conhecimento, a fim de sugerir pesquisas futuras e analisar o tema em estudo (BOTELHO; CUNHA; MACEDO, 2011).

A investigação foi realizada em cinco etapas (WHITTEMORE; KNAFL, 2005, p. 549): 1) identificação da questão norteadora, estabelecimento dos critérios de inclusão e exclusão dos artigos e busca nas bases de dados para identificação dos estudos; 2) categorização dos estudos e extração dos dados; 3) avaliação dos estudos incluídos na revisão; 4) discussão e interpretação dos resultados; e 5) síntese das informações evidenciadas nos artigos.

A questão orientadora desta pesquisa foi a seguinte: Quais são os softwares de Educação Alimentar e Nutricional utilizados ou desenvolvidos para o público idoso? Esta revisão foi motivada pelo desenvolvimento do estado de arte de uma dissertação em gerontologia.

Os critérios de inclusão definidos foram: artigos originais que respondessem à questão norteadora dessa revisão, disponíveis na íntegra, publicados em português, inglês ou espanhol, nos anos de 2009 a 2018. Escolheu-se este recorte temporal - últimos 10 anos - pois, segundo dados relativos à utilização do computador e da internet pelos idosos, houve um aumento crescente do uso das tecnologias a partir dos anos de 2010 e 2011 (DIAS, 2012). Foram excluídos desta pesquisa teses, dissertações, monografias, editoriais, artigos de revisão (narrativa, sistemática e integrativa), resumos de eventos, relatos de caso ou de experiência.

Editora Unijuí - Revista Contexto \& Saúde - ISSN 2176-7114 - v. 21, n. 43, jul./set. 2021 
A busca e seleção dos artigos foi realizada nas seguintes bases de dados: Medical Literature Analysis and Retrieval System Online (Medline), Literatura Latino-Americana e do Caribe em Ciências da Saúde (Lilacs) e Scopus, por dois avaliadores de forma independente, e, no caso de discordância na inclusão de algum estudo, um terceiro revisor foi consultado. A estratégia de busca foi elaborada com a utilização do Medical Subject Headings (MeSH) e dos Descritores em Ciências da Saúde (DeCS) e com o emprego dos operadores booleano AND e OR, que resultaram nas seguintes combinações descritas no Quadro 1.

Quadro 1 - Estratégia de busca

\begin{tabular}{|l|l|l|}
\hline Termos & $\begin{array}{c}\text { Base de } \\
\text { dados }\end{array}$ & \multicolumn{1}{c|}{ Estratégia de Busca } \\
\hline MESH & Medline & $\begin{array}{l}\text { (software OR mobile applications OR information technology) AND } \\
\text { (education OR educational technology) AND (food OR food quality } \\
\text { OR eating OR feeding behavior OR healthy food OR nutrition OR } \\
\text { diet) AND (aged OR aging OR elderly) }\end{array}$ \\
\hline DeCS & Lilacs & $\begin{array}{l}\text { Sofftware OR aplicativo móvel OR tecnologia da informação AND } \\
\text { (softmobile applications" OR "information technology" } \\
\text { quality" OR eating OR "feeding behavior" OR "healthy food" OR } \\
\text { nutrition OR diet AND aged OR aging OR elderly) }\end{array}$ \\
\hline $\begin{array}{l}\text { Educação OR tecnologia educacional OR educação alimentar OR } \\
\text { educação nutricional AND alimento OR qualidade dos alimentos } \\
\text { OR alimentação OR comportamento alimentar OR alimentação } \\
\text { saudável OR nutrição OR dieta AND idoso OR envelhecimento }\end{array}$ \\
\hline
\end{tabular}

Fonte: As autoras (2019).

O quantitativo de publicações encontrado nesta revisão foi determinado pelo cruzamento entre os descritores controlados. Como resultado, 320 publicações foram identificadas, mas foram excluídas 56 por duplicação, restando 264 para leitura dos títulos e, pelo fato de não contemplarem palavras ou ideias correlacionadas à temática do estudo, foram excluídas 195 publicações, restando 69 artigos para análise dos resumos. A partir dessa análise foram excluídos 24 artigos, resultando em 45 pré-selecionados para a leitura na íntegra. Após a verificação criteriosa, em resposta à questão de estudo dessa revisão e aos critérios de inclusão estabelecidos, 33 artigos foram excluídos e 12 foram submetidos à análise do rigor metodológico, segundo os critérios do Critical Appraisal Skills Programme (CASP, 2014), a partir do qual três artigos foram excluídos. A amostra final, portanto, foi composta por nove artigos, conforme Figura 1. 
Figura 1 - Descrição das etapas de seleção dos artigos incluídos na revisão integrativa

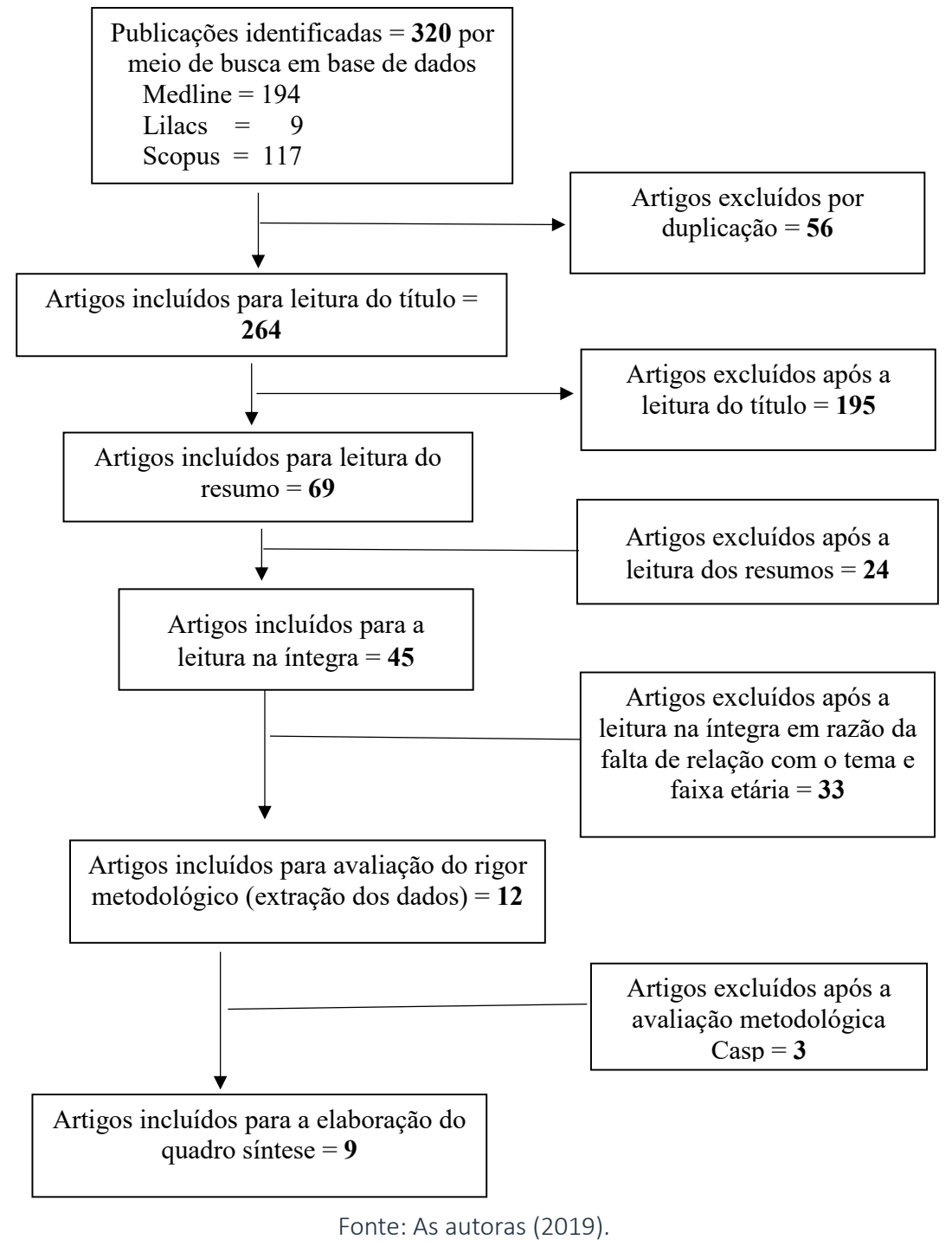

Na etapa de extração dos dados foi elaborado um quadro com os principais elementos dos estudos, constando os seguintes itens: autores, ano, país do estudo, objetivo, tipo de estudo, tecnologia utilizada e resultados alcançados (Quadro 2).

Já para a avaliação do rigor metodológico das publicações foi utilizado o instrumento do Critical Appraisal Skills Programme (Casp) com um checklist de dez itens pontuáveis (máximo 10 pontos), consistindo nas seguintes questões: objetivos do estudo, adequação do desenho metodológico ao tipo de estudo, coerência do desenho metodológico e a definição dos participantes, detalhamento da coleta de dados, relação entre pesquisador e pesquisados, cumprimento das considerações éticas, rigor na análise dos dados, clareza na apresentação dos resultados e importância da pesquisa. De acordo com a pontuação alcançada foram, então, classificados em duas categorias: A e B. Artigos com escore entre dez e seis pontos pertencem à categoria $A$, considerados estudos de boa qualidade metodológica e viés reduzido, enquanto os da categoria B são estudos com qua- 
lidade metodológica satisfatória, mas com potencial de viés aumentado, os quais alcançam o mínimo de cinco pontos (CASP, 2014), apresentados no Quadro 2. Aqueles com pontuação abaixo de cinco pontos foram excluídos.

Todos os estudos sintetizados no Quadro 2 referem-se a softwares de alimentação e nutrição, mas nem todos foram desenvolvidos exclusivamente para esta finalidade, ou seja, constava em um dos seus componentes a temática da educação alimentar e nutricional. Em vista deste fato, visando a organizar a interpretação dos resultados e a direcionar a discussão, os mesmos foram agrupados em duas categorias de abordagens de educação alimentar e nutricional (Figura 2): promoção da saúde e monitoramento de doenças.

\section{RESULTADOS}

Dos nove artigos incluídos na revisão integrativa (Quadro 1), seis haviam sido publicados nos últimos cinco anos e quatro foram realizados nos Estados Unidos.

Cinco deles (COOK et al., 2015; VANOH et al., 2018; JONGSTRA et al., 2017; ALl et al., 2012; WONG et al., 2012) tinham como foco a abordagem de Educação Alimentar e Nutricional na promoção da saúde, enquanto quatro estudos (WAKI et al., 2015; WU et al., 2015; RADHAKRISHNAN et al., 2016; YEHLE et al., 2012) focavam no monitoramento de doenças (Figura 2). Como promoção à saúde considerou-se aqueles sobre as modificações do estilo de vida como dieta, exercícios físicos, massa corporal, stress, tabagismo e medicação, enquanto a abordagem de Monitoramento de doenças compreendeu os cuidados de pacientes portadores de diabetes mellitus, câncer e doenças cardíacas.

Quadro 2 - Síntese de artigos referentes a softwares de EAN voltados para a população idosa

\begin{tabular}{|c|c|c|c|c|c|}
\hline $\begin{array}{l}\text { Autores } \\
\text { Ano } \\
\text { País de publi- } \\
\text { cação }\end{array}$ & Objetivos & $\begin{array}{l}\text { Tipo de estu- } \\
\text { do/amostra }\end{array}$ & Tecnologia & Resultados & Casp \\
\hline $\begin{array}{l}\text { Vanoh et al. } \\
2018 \\
\text { USA }\end{array}$ & $\begin{array}{l}\text { Desenvolver um } \\
\text { site para educar } \\
\text { os idosos sobre as } \\
\text { estratégias de modi- } \\
\text { ficação do estilo de } \\
\text { vida para retardar } \\
\text { o declínio cognitivo } \\
\text { leve. } \\
\text { Ferramenta de tria- } \\
\text { gem (dieta, saúde, } \\
\text { qualidade de vida, } \\
\text { trabalho e ativida- } \\
\text { des relacionadas ao } \\
\text { estilo de vida), guias } \\
\text { para melhoria da } \\
\text { memória, diário de } \\
\text { saúde e guia para } \\
\text { cardápios saudáveis. }\end{array}$ & $\begin{array}{l}\text { Descritivo } \\
\text { Amostra: } 73 \\
\text { idosos e } 32 \\
\text { cuidadores. }\end{array}$ & $\begin{array}{l}\text { Ferramenta } \\
\text { educacional } \\
\text { baseada na } \\
\text { Webcom para } \\
\text { idosos, que } \\
\text { inclui tela sen- } \\
\text { sível ao toque, } \\
\text { maior tamanho } \\
\text { de fonte, íco- } \\
\text { nes maiores e } \\
\text { componentes } \\
\text { multimídia. } \\
\text { - texto, imagens } \\
\text { e vídeos. }\end{array}$ & $\begin{array}{l}\text { Foi reconhecido } \\
\text { por pessoas ido- } \\
\text { sas e cuidadores } \\
\text { como ferramen- } \\
\text { ta de fiscalização } \\
\text { e educação para } \\
\text { o estágio primi- } \\
\text { tivo de perda de } \\
\text { memória e usa- } \\
\text { bilidade. }\end{array}$ & A \\
\hline
\end{tabular}




\begin{tabular}{|c|c|c|c|c|c|}
\hline $\begin{array}{l}\text { Jongstra et al. } \\
2017 \\
\text { Holanda }\end{array}$ & $\begin{array}{l}\text { Avaliar a eficácia } \\
\text { de uma plataforma } \\
\text { ativa de interação } \\
\text { pela Internet para } \\
\text { melhorar o perfil de } \\
\text { risco cardiovascular } \\
\text { de idosos com risco } \\
\text { cardiovascular ele- } \\
\text { vado. }\end{array}$ & $\begin{array}{l}\text { Prospectivo } \\
\text { randomizado } \\
\text { (quase expe- } \\
\text { rimental) } \\
\text { Amostra: } \\
41 \text { idosos } \\
\text { (interven- } \\
\text { ção= } 29 \text { e } \\
\text { grupo con- } \\
\text { trole }=16 \text { ) }\end{array}$ & $\begin{array}{l}\text { Plataforma da } \\
\text { web chamada } \\
\text { Envelhecimento } \\
\text { Saudável por } \\
\text { meio do acon- } \\
\text { selhamento na } \\
\text { internet (Ha- } \\
\text { tice). } \\
\text { - uso de men- } \\
\text { sagens de acon- } \\
\text { selhamento } \\
\text { (treinadores } \\
\text { (coach). }\end{array}$ & $\begin{array}{l}\text { Por ser desen- } \\
\text { volvida especi- } \\
\text { ficamente para } \\
\text { idosos, mostrou-se } \\
\text { adequado às ne- } \\
\text { cessidades desta } \\
\text { faixa etária aos } \\
\text { indivíduos mais } \\
\text { velhos e com } \\
\text { tendência atual } \\
\text { de uma aborda- } \\
\text { gem mais perso- } \\
\text { nalizada e digital } \\
\text { na medicina. }\end{array}$ & $A$ \\
\hline $\begin{array}{l}\text { Radhakrishnan } \\
\text { et al. } \\
2016 \\
\text { USA }\end{array}$ & $\begin{array}{l}\text { Desenvolver e testar } \\
\text { o protótipo de um } \\
\text { jogo digital amigável } \\
\text { para melhorar o } \\
\text { conhecimento sobre } \\
\text { os medicamentos, } \\
\text { dieta e atividade } \\
\text { física, monitoramen- } \\
\text { to de sintomas de } \\
\text { idosos com insufici- } \\
\text { ência cardíaca }\end{array}$ & \begin{tabular}{|l|} 
Prospectivo \\
antes e após \\
(Quase-expe- \\
rimental) \\
Amostra: 26 \\
idosos com \\
insuficiência \\
cardíaca.
\end{tabular} & $\begin{array}{l}\text { Jogo digital } \\
\text { (Game) de ca- } \\
\text { ça-níqueis de } \\
\text { cassino } \\
\text { Aplicativo para } \\
\text { iOS. } \\
\text { Tablet } \\
\text { - Game }\end{array}$ & $\begin{array}{l}\text { Os idosos com } \\
\text { insuficiência } \\
\text { cardíaca acha- } \\
\text { ram satisfatório } \\
\text { e aceitável, pois } \\
\text { permitiu melhor } \\
\text { conhecimento } \\
\text { para o autoge- } \\
\text { renciamento da } \\
\text { doença. }\end{array}$ & $A$ \\
\hline $\begin{array}{l}\text { Cook et al. } \\
2015 \\
\text { USA }\end{array}$ & $\begin{array}{l}\text { Avaliar o impacto de } \\
\text { um programa multi- } \\
\text { mídia de promoção } \\
\text { da saúde baseado } \\
\text { na Web sobre as } \\
\text { atitudes e mudanças } \\
\text { positivas relaciona- } \\
\text { das a stress, dieta, } \\
\text { atividade física, } \\
\text { crenças sobre o } \\
\text { envelhecimento e } \\
\text { uso fumo. }\end{array}$ & $\begin{array}{l}\text { Ensaio clínico } \\
\text { randomizado } \\
\text { Amostra: } \\
278 \text { traba- } \\
\text { Ihadores de } \\
50 \text { anos ou } \\
\text { mais }\end{array}$ & $\begin{array}{l}\text { Programa de } \\
\text { multimídia } \\
\text { (vídeos, fotos } \\
\text { e gráficos) ba- } \\
\text { seado na Web } \\
\text { contendo infor- } \\
\text { mações e orien- } \\
\text { tação sobre } \\
\text { envelhecimento } \\
\text { saudável, dieta, } \\
\text { atividade física, } \\
\text { gerenciamento } \\
\text { do stress e uso } \\
\text { de fumo. } \\
\text { - Internet }\end{array}$ & $\begin{array}{l}\text { Potencial de } \\
\text { contribuição às } \\
\text { práticas dieté- } \\
\text { ticas e de exer- } \\
\text { cícios de curto } \\
\text { prazo aos idosos } \\
\text { que trabalham, } \\
\text { sendo as melho- } \\
\text { rias observadas } \\
\text { principalmente } \\
\text { entre as mulhe- } \\
\text { res. }\end{array}$ & $A$ \\
\hline $\begin{array}{l}\text { Waki et al. } \\
2015 \\
\text { Japão }\end{array}$ & $\begin{array}{l}\text { Testar uma versão } \\
\text { mais próxima do } \\
\text { DialBetics (Monito- } \\
\text { ramento do diabe- } \\
\text { tes). }\end{array}$ & $\begin{array}{l}\text { Tipo de es- } \\
\text { tudo: Ensaio } \\
\text { clínico ran- } \\
\text { domizado. } \\
\text { Amostra: } 22 \\
\text { pacientes } \\
\text { com DM. }\end{array}$ & $\begin{array}{l}\text { DialBetics com } \\
\text { FoodLog é um } \\
\text { aplicativo para } \\
\text { smartphone } \\
\text { para registro } \\
\text { alimentar e pro- } \\
\text { cessamento de } \\
\text { foto da refeição, } \\
\text { para avaliação e } \\
\text { aconselhamen- } \\
\text { to nutricional } \\
\text { em tempo real } \\
\text { para controle do } \\
\text { diabetes. }\end{array}$ & $\begin{array}{l}\text { A ferramenta } \\
\text { foi considerada } \\
\text { eficaz e con- } \\
\text { veniente, pois } \\
\text { conta com um } \\
\text { registro alimen- } \\
\text { tar multimídia } \\
\text { com entrada de } \\
\text { foto de refeição, } \\
\text { proporcionan- } \\
\text { do suporte em } \\
\text { tempo real para } \\
\text { modificação da } \\
\text { dieta. }\end{array}$ & $A$ \\
\hline
\end{tabular}




\begin{tabular}{|c|c|c|c|c|c|}
\hline $\begin{array}{l}\text { Wu et al. } \\
2015 \\
\text { Taiwan }\end{array}$ & $\begin{array}{l}\text { Desenvolver e testar } \\
\text { uma intervenção } \\
\text { assistida por com- } \\
\text { putador pessoal } \\
\text { (PC) para acelerar a } \\
\text { recuperação estado } \\
\text { nutricional de pa- } \\
\text { cientes com câncer } \\
\text { pós-gastrectomia. }\end{array}$ & \begin{tabular}{|l|} 
Prospectivo, \\
randomizado \\
(Quase expe- \\
rimental) \\
Amostra= \\
40 pacien- \\
tes: Grupo \\
App $(n=20)$, \\
grupo con- \\
trole $(n=20)$. \\
Acima de 20 \\
anos,
\end{tabular} & $\begin{array}{l}\text { Aplicativo para } \\
\text { tablet. } \\
\text { - mensagens de } \\
\text { texto }\end{array}$ & $\begin{array}{l}\text { Mostrou ser uma } \\
\text { solução viável } \\
\text { para o desafio } \\
\text { de informações } \\
\text { pós-operatórias } \\
\text { de autorregistro } \\
\text { e uma alterna- } \\
\text { tiva ferramenta } \\
\text { de autocuidado } \\
\text { para pacientes } \\
\text { com câncer } \\
\text { gástrico. }\end{array}$ & $B$ \\
\hline $\begin{array}{l}\text { Ali et al. } \\
2012 \\
\text { Malásia }\end{array}$ & $\begin{array}{l}\text { Desenvolver um } \\
\text { pacote digital de } \\
\text { educação nutricio- } \\
\text { nal (diretrizes para } \\
\text { a alimentação sau- } \\
\text { dável, questionário, } \\
\text { calculadora de índi- } \\
\text { ce de massacorporal } \\
\text { (IMC) e vídeo de } \\
\text { demonstração de } \\
\text { exercícios) e avaliar } \\
\text { sua aceitação entre } \\
\text { pessoas idosas. }\end{array}$ & \begin{tabular}{|l|} 
Descritivo \\
(usabilidade) \\
Amostra: 31 \\
idosos.
\end{tabular} & $\begin{array}{l}\text { Plataforma de } \\
\text { educação nu- } \\
\text { tricional digital } \\
\text { - We Sihat - } \\
\text { uma tecnologia } \\
\text { touch-screen } \\
\text { - combina tex- } \\
\text { to, áudio, vídeo } \\
\text { e gráficos. }\end{array}$ & $\begin{array}{l}\text { Avaliação po- } \\
\text { sitiva, com } \\
\text { preferência do } \\
\text { usuário para } \\
\text { o uso de um } \\
\text { pacote digital e } \\
\text { interativo com } \\
\text { uma interface de } \\
\text { tela de toque. O } \\
\text { design direcio- } \\
\text { nado às pessoas } \\
\text { idosas contribuiu } \\
\text { para a aceitabili- } \\
\text { dade e interação } \\
\text { com o sistema } \\
\text { na compreensão } \\
\text { sobre dietas } \\
\text { saudáveis. }\end{array}$ & $A$ \\
\hline $\begin{array}{l}\text { Wong et al. } \\
2012 \\
\text { Taiwan }\end{array}$ & $\begin{array}{l}\text { Avaliar a intenção de } \\
\text { uso do lembrete de } \\
\text { medicação doutor. } \\
\text { onipresente, toque } \\
\text { compartilhado e } \\
\text { relógio inteligente, } \\
\text { que integram o } \\
\text { sistema de Cuidados } \\
\text { Interativo, Compre- } \\
\text { ensivo e Inteligente } \\
\text { (ICIC) para idosos, } \\
\text { usando um modelo } \\
\text { de aceitação tecno- } \\
\text { lógica } \\
\text { Modificada (TAM). }\end{array}$ & $\begin{array}{l}\text { Descritivo } \\
\text { Amostra: } 121 \\
\text { idosos. }\end{array}$ & \begin{tabular}{|l|} 
Plataforma digi- \\
tal com serviços \\
de cuidados ao \\
idoso, conten- \\
do: lembrete \\
de medicação, \\
consulta mé- \\
dica, relógio \\
inteligente que \\
monitora as \\
condições psico- \\
lógicas dos ido- \\
sos, que integra \\
um eletrocar- \\
diograma, hora \\
e notificações \\
- usa fotos, \\
vídeo, música e \\
documentos. \\
\end{tabular} & \begin{tabular}{|l} 
O sistema teve \\
boa aceitação \\
da população \\
idosa, pois ofe- \\
rece uma inter- \\
face amigável \\
de solução em \\
atendimento e \\
monitoramento \\
da telemedicina, \\
melhorando a \\
interação social \\
e qualidade do \\
cuidado à popu- \\
lação idosa.
\end{tabular} & $A$ \\
\hline $\begin{array}{l}\text { Yehle et al. } \\
2012 \\
\text { USA }\end{array}$ & $\begin{array}{l}\text { Examinar os de- } \\
\text { safios dietéticos } \\
\text { dos pacientes com } \\
\text { doença coronariana } \\
\text { e as estratégias } \\
\text { para lidar com estes } \\
\text { desafios, bem como } \\
\text { a viabilidade da } \\
\text { utilização de uma } \\
\text { tecnologia baseada } \\
\text { na Web para auxiliar } \\
\text { nas escolhas alimen- } \\
\text { tares. }\end{array}$ & $\begin{array}{l}\text { Descritivo } \\
\text { Amostra: } 20 \\
\text { pacientes } \\
\text { com doença } \\
\text { coronariana } \\
\text { e } 7 \text { cuidado- } \\
\text { res. }\end{array}$ & $\begin{array}{l}\text { O Food for the } \\
\text { Heart (FFH) e o } \\
\text { Mobile Magic } \\
\text { Lens (MML), } \\
\text { foram desenvol- } \\
\text { vidos para aju- } \\
\text { dar nas escolhas } \\
\text { diárias da dieta, } \\
\text { rastrear a ade- } \\
\text { rência à dieta, } \\
\text { sugerir lista de } \\
\text { compras, recei- } \\
\text { tas e reúne uma } \\
\text { comunidade } \\
\text { on-line para } \\
\text { suporte, troca } \\
\text { de informações } \\
\text { e receitas. }\end{array}$ & $\begin{array}{l}\text { Os pacientes } \\
\text { com doença } \\
\text { cardíaca corona- } \\
\text { riana conside- } \\
\text { raram valiosos } \\
\text { na escolha de } \\
\text { alimentos e na } \\
\text { adesão à dieta, } \\
\text { especialmente } \\
\text { se característi- } \\
\text { cas específicas } \\
\text { forem adicio- } \\
\text { nadas para dar } \\
\text { assistências aos } \\
\text { pacientes que } \\
\text { desejam fazer } \\
\text { modificações. }\end{array}$ & B \\
\hline
\end{tabular}


Figura 2 - Categorização das publicações incluídas na revisão integrativa, segundo abordagem do aplicativo com contribuição para a educação alimentar e nutricional de idosos no período de 2009 a 2018

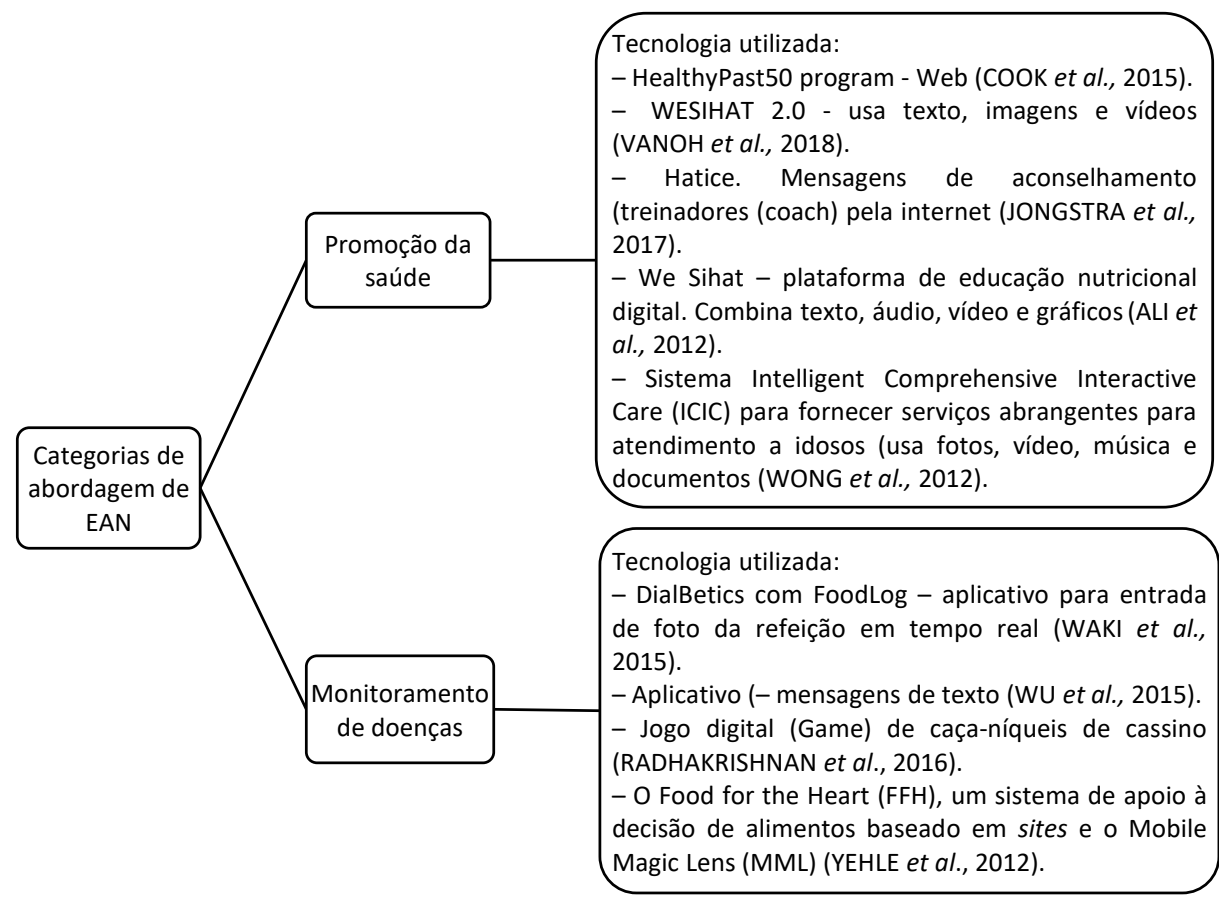

Fonte: As autoras (2019).

\section{DISCUSSÃO}

A propensão acelerada de recursos de tecnologia, informação e comunicação na área da saúde e a ampliação da aquisição de smartphones entre as pessoas idosas, potencializa o uso dos aplicativos móveis voltados à gerontologia (AMORIM et al., 2018). O desenvolvimento de softwares voltados à população idosa visando à educação alimentar e nutricional, no entanto, é ainda incipiente, embora seja de grande importância como uma ferramenta que aproxima o idoso das tecnologias de informação e comunicação, desde que seja planejada para atender algumas dificuldades decorrentes das alterações fisiológicas do envelhecimento.

Os achados deste estudo mostraram que programas com apoio de softwares de educação alimentar e nutricional são ferramentas potenciais na promoção da saúde e no monitoramento de enfermidades. As principais características encontradas nestes aplicativos se referiam a modificações do estilo de vida, incluindo, principalmente, a EAN quanto à dieta saudável, cardápios, ingestão de frutas e verduras e controle do peso corporal, acompanhadas da orientação na prática de atividades físicas, na abordagem de promoção de saúde e, também, no monitoramento de doenças.

Percebeu-se que a maioria deles $(66,7 \%)$ tinha alguma relação com enfermidades, mesmo aqueles categorizados com abordagem na promoção da saúde $(n=5)$, considerando que dois deles, Vanoh et al. (2018) e Jongstra et al. (2017), tinham como meta a promoção da saúde com EAN envolvida para evitar declínio 
cognitivo e diminuir risco cardiovascular em pacientes de alto risco. Na revisão de Prado, Vilaça e Silva (2020) sobre o uso de aplicativos móveis que estimulem a prática da alimentação saudável pela população acima de 65 anos, não foram encontrados estudos sobre o uso, nesta faixa etária, de tecnologias digitais com foco no estímulo aos hábitos alimentares saudáveis sem necessariamente relacioná-los a uma patologia preexistente, evidenciando a dificuldade em se desenvolver aplicativos móveis inteligentes que atinjam essa população.

Cook et al. (2015), que avaliaram o impacto de um programa multimídia denominado HealtyPast50 entre trabalhadores de 50 anos ou mais na promoção da saúde, constataram que o grupo que teve acesso ao programa mostrou meIhora significativamente maior nos construtos-chave de saúde durante o período de teste de três meses, comparado àqueles do grupo de controle, especialmente sobre a autoeficácia de mudança de comportamento, planejamento de alimentação saudável e exercícios leves. Embora o programa não fosse exclusivo para idosos, ele teve seu desenvolvimento voltado para o envelhecimento.

Com o objetivo de desenvolver um site para educar os idosos sobre as estratégias de modificação do estilo de vida para retardar o declínio cognitivo leve, denominado WESIHAT 2.0, Vanoh et al. (2018) consideraram várias etapas no desenvolvimento do software para atender as alterações fisiológicas que ocorrem com o envelhecimento, como tela sensivel ao toque, maior tamanho de fonte, ícones maiores e componentes multimídia apresentados na forma de texto, imagens e vídeos. Para os idosos e cuidadores, esta ferramenta foi adequada para identificar sinais precoces de alterações cognitivas leves, avaliados por um instrumento de triagem de memória, e as informações oferecidas foram reconhecidas como importantes para aumentar o conhecimento dietético e de saúde entre estes.

Também, um estudo avaliou o desenvolvimento e validação de uma plataforma especialmente projetada para o Envelhecimento Saudável por meio de mensagens de aconselhamento (coach) pela internet ao idoso (Hatice), por Jongstra et al. (2017), que mostraram ser eficaz para treinar/motivar os participantes em sua vida para melhorar o perfil de risco cardiovascular de idosos com este risco elevado. A intervenção centrou-se em sete riscos cardiovasculares modificáveis: hipertensão, dislipidemia, diabetes mellitus, excesso de peso, inatividade física, tabagismo e nutrição saudável.

Uma plataforma especificamente de educação nutricional digital para idosos foi desenvolvida e testata por Ali et al. (2012). Os participantes do estudo meencionaram que o pacote nutricional digital (We Sihat), desenvolvido para idosos, utilizou imagens adequadas, combinações atraentes de cor e tamanhos apropriados, o que possibilitou o fácil entendimento das recomendações dietéticas, que foram escritas usando frases simples e breves.

Wong et al. (2012) avaliaram a intenção de uso de quatro componentes de um sistema interativo, compreensivo e inteligente de cuidados (Icic) para idosos, visando a fornecer atendimento e monitoramento por meio de telemedicina. Embora a avaliação tenha sido feita por pessoas acima de 41 anos, o sistema foi desenvolvido para a população idosa e esta apresentou boa aceitação da tecnologia pois forneceu um viável modelo interdisciplinar integrando cuidados médicos, dieta, vida, transporte, educação, entretenimento e demandas de segurança.

Editora Unijuí - Revista Contexto \& Saúde - ISSN 2176-7114 - v. 21, n. 43, jul./set. 2021 
Em seu estudo, Waki et al. (2015) testaram por uma semana um aplicativo por smartphone denominado DialBetics, observando diminuição dos níveis bioquímicos da hemoglobina glicada. Este aplicativo consistia no registro alimentar multimídia com a entrada de fotos de refeições, fornecendo ao paciente suporte em tempo real para controle do diabetes. Este sistema foi considerado uma ferramenta positiva em razão do rápido retorno da avaliação dietética para os pacientes, permitindo mais rapidamente modificar seus hábitos de dieta com base nas fotos. Também, a avaliação fotográfica da dieta possibilitou tanto aos pacientes com diabetes quanto aos profissionais de saúde verificar se ocorreram ou não mudanças na dieta ao longo do tempo. Resultado similar foi encontrado no estudo de Boels et al. (2018), que avaliou autogerenciamento dos pacientes com diabetes mellitus tipo $2 \mathrm{com}$ insulinoterapia, tendo como resultado a melhora dos níveis de hemoglobina (HbA1c level $\leq 53 \mathrm{mmol} / \mathrm{mol}$ ( $\leq 7 \%$ ) sem hipoglicemia.

O estudo de Wu et al. (2015) desenvolveu e testou uma intervenção assistida por computador pessoal (PC) com relação ao estado nutricional, para acelerar a recuperação de pacientes com câncer pós-gastrectomia. O sistema facilitado por este aplicativo, com mensagens de texto, informava tanto os pacientes quanto os profissionais da área médica quanto à perda de peso corporal acima de $5 \%$ aos três meses após a cirurgia, indicando que o paciente poderia requerer uma avaliação nutricional e uma intervenção como consulta com um nutricionista para recuperar a condição de risco nutricional ou desnutrição. Os resultados indicaram que o grupo intervenção (uso de aplicativos) teve uma porcentagem menor de perda de peso corporal em relação ao grupo controle durante um período de acompanhamento de 6 meses, que pode ser decorrente do maior número de consultas ambulatoriais realizadas.

No estudo de Radhakrishnan et al. (2016) foi desenvolvido e testado um protótipo de jogo digital no estilo caça-níqueis de cassino, com o objetivo de meIhorar o conhecimento bem como o gerenciamento do comportamento de idosos com insuficiência cardíaca (IC). Para tanto, o jogo trouxe informações sobre a doença, medicações, modificações na dieta e atividade física, e, ainda, verificação de sintomas. Os comentários positivos indicaram principalmente a utilidade do jogo em fornecer dicas, lembretes e informações para aqueles com menor escolaridade ou com um diagnóstico recente de IC.

O Food for the Heart (FFH), um sistema de apoio à decisão de alimentos baseado em sites, e o Mobile Magic Lens (MML), um sistema baseado em dispositivos móveis, foram desenvolvidos por Yehle et al. (2012) para avaliar os desafios dietéticos e estratégias para a adesão em pacientes com doença coronariana bem como a viabilidade da utilização de tecnologia para auxiliar nas escolhas alimentares adequadas diariamente. Cinco temas emergiram do estudo: os desafios dietéticos mais comuns foram a redução da ingestão de carboidratos e o controle de porções; o apoio clínico e social facilita a adesão à dieta; o site da Food for the Heart (FFH) pode facilitar o planejamento das refeições, embora apontaram para a necessidade de recursos adicionais, como a inclusão de uma aba para comunicação com os profissionais de saúde; o Mobile Magic Lens (MML) é fácil de usar, mais rápido do que ler rótulos e ajudaria os pacientes a fazer escolhas saudáveis.

Editora Unijuí - Revista Contexto \& Saúde - ISSN 2176-7114 - v. 21, n. 43, jul./set. 2021 
Ações educativas são estratégias de promoção de saúde e contribuem na manutenção do papel social do idoso trazendo, além do conhecimento, um resgate da autoestima e autonomia, tornando o sujeito ativo e participativo. Além disso, a inserção da maturidade na educação possibilita uma melhor compreensão das transformações do mundo, favorecendo a interação entre as gerações (GARCIA et al., 2019).

\section{CONSIDERAÇÕES FINAIS}

Como conclusão, observou-se que a educação em saúde, quando associada às tecnologias para a população idosa, em cinco dos artigos selecionados parece ser uma ferramenta auxiliar para o autocuidado e a corresponsabilização do cuidado no que se refere à abordagem de promoção à saúde de educação alimentar e nutricional para a população em estudo.

Esta revisão integrativa identificou estudos sobre o desenvolvimento de softwares de educação alimentar e nutricional de abordagem na promoção da saúde, orientando sobre mudanças do estilo de vida por meio de uma dieta adequada, controle de peso e de exercícios físicos e de abordagem de monitoramento de doenças, como diabetes mellitus, câncer e doenças cardíacas.

Torna-se relevante comentar que não foram encontrados estudos desenvolvidos no Brasil que pudessem ser incluídos na pesquisa, indicando a necessidade em trabalhos futuros. Deste modo, espera-se contribuir para o campo de conhecimento de desenvolvimento de softwares, especialmente de educação alimentar e nutricional voltados à população idosa, a fim de promover informação de qualidade e entretenimento a esse grupo etário.

A EAN tem nos aplicativos um grande alicerce, para além da promoção da afirmação e da capacidade dos idosos quanto à melhoria da alimentação saudável e autogestão da saúde, servindo como um instrumento de acompanhamento de consulta nutricional e esclarecimentos para familiares, de forma geral.

\section{REFERÊNCIAS}

ALI, N. M. et al. Design of an interactive digital nutritional education package for elderly people. Informatics for Health and Social Care, Bangi Selangor, v. 37, n. 4, p. 217-229, 2012.

AMORIM, D. N. P. et al. Aplicativos móveis para a saúde e o cuidado de idosos. Reciis Rev. Eletron. comum. Inf. Inov. Saúde, v. 12, n. 1, p. 5-71, 2018.

BOELS, A. M. et al. Effectiveness of diabetes self-management education via a smartphone application in insulin treated type 2 diabetes patients - design of a randomised controlled trial (TRIGGER study). BMC Endocrine Disorders, v. 18, n. 1, p. 74-83, 2018.

BOTELHO, L. L. R.; CUNHA, C. C. A.; MACEDO, M. O método da revisão integrativa nos estudos organizacionais. Gestão e Sociedade, v. 5, n. 11, p. 121-136, 2011.

BRAZ, V. N.; LOPES, B. M. Evaluation of mobile applications related to nutrition. Public Health Nutrition, v. 22, n. 7, p. 1.209-1.214, 2018.

CASP. Critical Appraisal Skills Programme. Casp Checklists (URL used). Oxford [internet]. 2014. Disponível em: http://www.casp-uk.net/checklists. Acesso em: 12 jan. 2019. 
COOK, R. F. et al. A Web-Based Health Promotion Program for Older Workers: Randomized Controlled Trial. Journal of medical internet research, Alexandria, v. 17, n. 3, p. 1-16, 2015.

COSTA, E. O.; BIFANO, A. C. S. Idosos e tecnologias: uma pesquisa bibliográfica. Estud. Interdiscipl. Envelhec., Porto Alegre, v. 22, n. 2, p. 113-131, 2017.

DIAS, I. O uso das tecnologias digitais entre os seniores - motivações e interesses. Sociologia, Problemas e Práticas, n. 68, p. 51-77, 2012. DOI: 10.7458/SPP201268693

GARCIA, K. R. et al. Inserção de pessoas na maturidade na educação superior. Revista de Divulgação Científica Sena Aires, v. 8, n. 2, p. 132-138, 2019.

GUIMARÃES, H. N. A importância das tecnologias de informação e comunicação na vida social dos idosos. 2017. Trabalho (Conclusão de Curso) - Universidade Estadual da Paraíba, Patos, Paraíba, 2017.

IBGE. Instituto Brasileiro de Geografia e Estatística. 2016. Síntese de indicadores sociais: uma análise das condições de vida da população brasileira. Rio de Janeiro, 2016. 146 p. Disponível em: https://biblioteca.ibge.gov.br/visualizacao/livros/liv98965.pdf. Acesso em: 23 set. 2019.

JONGSTRA, S. et al. Development and Validation of an Interactive Internet Platform for Older People: The Healthy Ageing Through Internet Counselling in the Elderly Study. Telemedicine and e-Health, Amsterdam, v. 23, n. 2, p. 1-9, 2017.

MENDEZ, C. B. et al. Mobile educational follow-up application for patients with peripheral arterial disease. Rev. Latino-Am. Enfermagem, 27, p. e3122, 2019.

PADRÃO, S. M.; AGUIAR, O. B.; BARÃO, G. O. D. Educação alimentar e nutricional: a defesa de uma perspectiva contra-hegemônica e histórico-crítica para educação. Demetra: Alimentação, Nutrição \& Saúde, v. 12, n. 3, p. 665-682, 2017.

PRADO, A. R. C.; VILAÇA, K. H. C.; SILVA, G. A. C. Aplicativos móveis para a promoção de hábitos saudáveis de alimentação em idosos. Revista RGCTI, v. 4, n. 1, p. 11-20, 2020.

RADHAKRISHNAN, K. et al. Interactive Digital e-Health Game for Heart Failure Self-Management: A Feasibility Study. Games for Health Journal: Research, Development, and Clinical Applications, Austin, v. 5, n. 6, p. 366-374, 2016.

SANTOS, R. F.; ALMÊDA, K. A. O envelhecimento humano e a inclusão digital: análise do uso das ferramentas tecnológicas pelos idosos. Ci. Inf. Rev., v. 4, n. 2, p. 59-68, 2017.

$\mathrm{VANOH}$, D. et al. Development and assessment of a web-based intervention for educating older people on strategies promoting healthy cognition. Clinical Interventions in Aging, Kuala Lumpur, v. 13, p. 1.787-1.798, 2018.

WAKI, K. et al. DialBetics with a multimedia food recording tool, FoodLog: Smartphone-Based Self-Management for Type 2 Diabetes. Journal of Diabetes Science and Technology, v. 9, n. 3, p. 534-540, 2015.

WHITTEMORE, R.; KNAFL, K. The integrative review: updated methodology. J Adv Nurs., v. 52, n. 5, p. 546-553, 2005.

WONG, A. M. K. et al. Technology Acceptance for an Intelligent Comprehensive Interactive Care (ICIC) System for Care of the Elderly: A Survey-Questionnaire Study. PLoS ONE, v. 7, p. 1-7, 2012.

WU, J. M. et al. Tablet PC-enabled application intervention for patients with gastric cancer undergoing gastrectomy. Computer methods and programs in biomedicine, Taipei, $\mathrm{p}$. 101-109, 2015.

YEHLE, K. S. et al. A qualitative analysis of coronary heart disease patient views of dietary adherence and web-based and mobile-based nutrition tools. J. Cardiopulm Rehabil Prev., Cedarville, v. 32, n. 4, p. 1-10, 2012. 